\title{
mano: A Wearable Hand Exoskeleton for Activities of Daily Living and Neurorehabilitation
}

\author{
Luca Randazzo, Iñaki Iturrate, Serafeim Perdikis and J. d. R. Millán
}

\begin{abstract}
Hand sensorimotor impairments are among the most common consequences of injuries affecting the central and peripheral nervous systems, leading to a drastic reduction in the quality of life for affected individuals. Combining wearable robotic exoskeletons and human-machine interfaces is a promising avenue for the restoration and substitution of lost and impaired functions for these users. In this study, we present a novel hand exoskeleton, mano, designed to assist and restore hand functions of people with motor disabilities during activities of daily living (ADL) and in neurorehabilitative scenarios. Compared to state-of-the-art devices, our system is fully wearable, portable and minimally obtrusive on the hand. The exoskeleton can actively control flexion and extension of all fingers, while allowing natural somatosensorial interactions with the environment surrounding the users. We evaluated the device from four different perspectives. A mechanical characterization, showing that the exoskeleton can cover more than $70 \%$ of healthy hand workspace and it can achieve forces at the fingertips sufficient for ADL. A functional characterization, where we showed how two users who suffered from spinal cord injuries were able to perform several ADL for the first time since their accidents. Thirdly, we evaluated the system from a neuroimaging perspective, showing that the device can elicit EEG brain patterns typical of natural hand motions. We finally exemplified the control of the hand exoskeleton within an exemplar framework, a brain-machine interface scenario, showing how motor intention can be successfully decoded for a continuous control of the device. Overall, our results showed that the device represents an ecological solution for use both in ADL and in scenarios aimed at promoting sensorimotor recovery.
\end{abstract}

Index Terms-Prosthetics and exoskeletons, rehabilitation robotics, wearable robots, brain-machine interface.

\section{INTRODUCTION}

$\mathbf{O}$ UR hands play a fundamental role throughout the course of our lives, mediating some of the most distinctive skills of humans: the ability to interact and shape the world around us. Hand motor and sensory functions, though, can be severely impaired due to injuries affecting the central and peripheral nervous systems and the musculoskeletal apparatus, such as cerebrovascular accidents, spinal cord injuries and myopathies. Hence, deficits in arms and hand functioning have a big impact on the overall quality of life and independence of affected individuals.

Manuscript received May 24, 2017; accepted October 3, 2017. Date of publication; date of current version. This letter was recommended for publication by Associate Editor F. Chen and Editor A. M. Okamura upon evaluation of the reviewers' comments. This work was supported by the Swiss NCCR Robotics. The work of I. Iturrate was supported by the "EPFL Fellows" program. (Corresponding author: Luca Randazzo)

The authors are with Defitech Chair in Brain-Machine Interface, Center for Neuroprosthetics, Ecole Polytechnique Fédérale de Lausanne, Lausanne 1015 , Switzerland (email: luca.randazzo@epfl.ch).

Digital Object Identifier 10.1109/LRA.2017.2771329

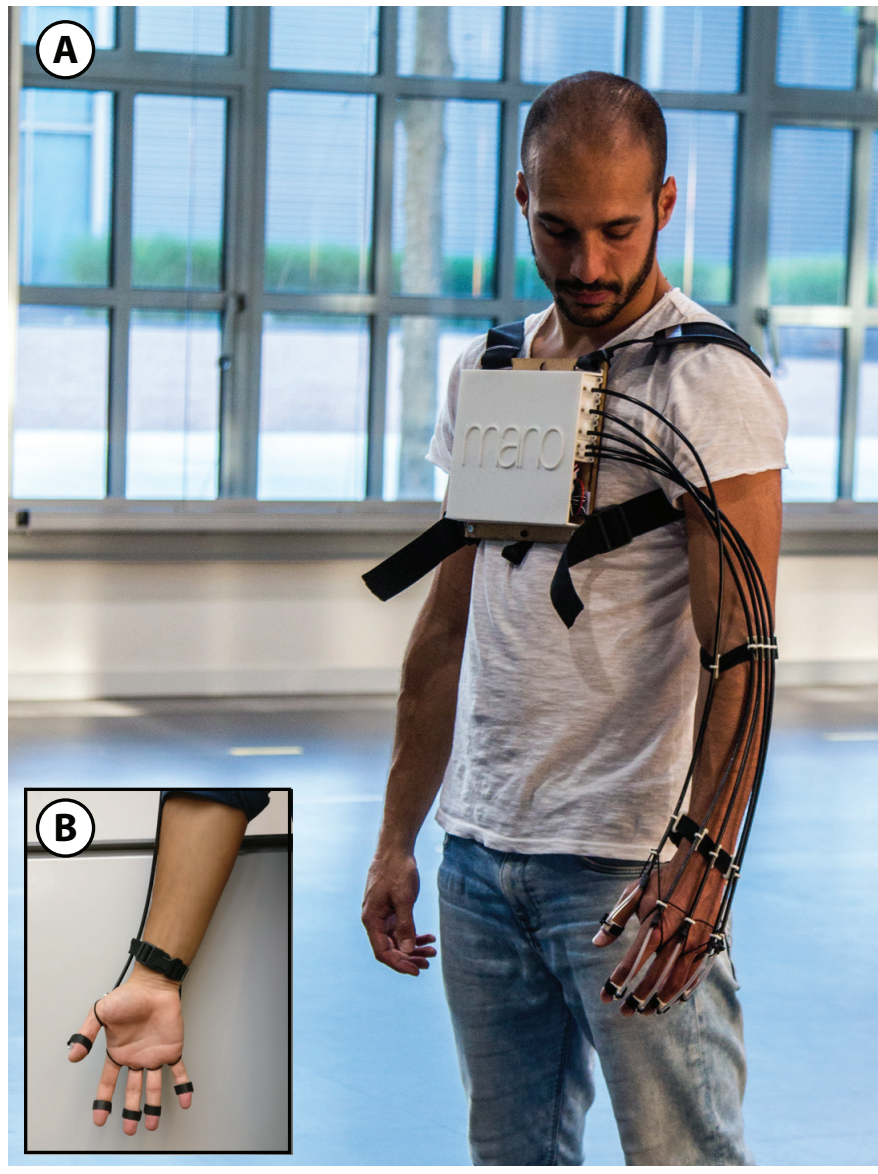

Fig. 1. The mano exoskeleton. The device consists of two main parts: a chest-pack (which hosts the actuation, energy storage and control units) and artificial tendons (which transmit the motion from the actuators to the fingers of the wearer). All the exoskeletal structures are placed on the hand dorsal part not to hamper somatosensation on palm and fingertips during use.

In recent years, the combined use of wearable robotic systems and human-machine interfaces has been proposed as a tool for complementing and restoring impaired functions in users with motor disabilities. By acting as transparent extensions of human bodies, these systems can translate movement intentions into control signals for wearable robotic devices, for the sake of providing motor assistance or promoting recovery of functions [1], [2].

However, despite these approaches are commonly used in research laboratories and highly-specialized centers, they still suffer from an important limitation: their adoption by users on a daily basis is limited because of complexity, poor usability and high costs. Indeed, the translation of these systems to domestic settings could enable intensive and continuous use 
during meaningful activities of daily living (ADL), ultimately improving their effectiveness [3].

In this study, we present a novel hand exoskeleton, mano (Figure 1), aimed at overcoming these limitations and at representing a viable solution for the assistance and restoration of hand motor functions. Section II presents the design process of the device, its characterization in terms of kinematics and achievable forces, and the results of qualitative tests with two users who suffered from spinal cord injuries. In Section III, we present a characterization of the exoskeleton from a neuroimaging perspective and the results of use within an exemplar human-machine interfacing framework, a brainmachine interfacing (BMI) scenario. Finally, in Section IV, we discuss the limitations of the current study as well as future directions worth investigation.

\section{MANO}

The mano device was designed with the main purpose of enabling intensive use during ADL by a wide range of users with motor disabilities, while preserving as much as possible patterns of natural interactions with these settings. Several studies have highlighted how ecological interactions with meaningful and relevant environments can lead to increased acceptance of assistive devices and to a better recovery with respect to therapies performed in hospital settings [4], [5]. To this end, we aimed at developing a device intensively usable in $\mathrm{ADL}$, both for assistive and neurorehabilitative purposes.

\section{A. Related work}

Many exoskeletal systems have been proposed for hand rehabilitation and assistance (see [6], [7], [8], [9] for comprehensive reviews). Here, we present a short overview of the devices we identified as possibly complying with requirements of use in ADL. In particular, in accordance with criteria related to wearability, wide usability and comfort, we focused on soft and lightweight exoskeletons, excluding systems based on hard or bulky structures on the hand and non-portable devices. The first were excluded because they are poorly adaptable to different hand sizes and tasks and because they tend to get entangled with external objects, tear clothes, etc., presenting serious limitations for viable daily use. Non-portable devices were excluded because they cannot be easily displaced and they are therefore incompatible with many ADL.

Reference systems emulate the natural functioning of human fingers by adopting tendon-driven mechanisms. These designs enable the self-alignment of the exoskeletal structure(s) to the user's hand without the use of complex mechanisms while allowing the remote placement of the actuation units with respect to the hands, to the benefit of soft and lightweight profiles on the arms. For example, the NASA RoboGlove [10] and Soft Extra Muscle [11] devices exploit artificial tendons to actively control fingers flexion. Similar concepts are used in the Soft Robotics Exoskeleton [12], in the Exo-Glove [13], and in the Exo-Glove Poly [14]. Although lightweight on the hands, these devices mainly suffer from hindering natural somatosensation on palms and fingertips or from the lack of control over both flexion and extension of fingers.
Several concepts have been proposed for preserving natural somatosensation during use. Polygerinos et al. [15] developed hydraulic-actuated soft muscles capable of controlling fingers flexion from the hand's dorsal side. Although relatively lightweight on the hand ( $285 \mathrm{~g})$, the actuation and control units of this system are rather heavy $(\sim 3.3 \mathrm{~kg})$, limiting the overall portability and usability in ADL. The Gloreha glove [16] is a commercial device which exploits bowden cables in a push-pull configuration to control both flexion and extension of fingers from the hand's dorso. Similarly to the previous device, this system has the advantage of being lightweight on the hand $(\sim 50 \mathrm{~g})$ but relies on bulky actuation and control units $(\sim 5 \mathrm{~kg})$ which make it non-portable. Additionally, due to the anchoring points of the exoskeletal structures, these systems hamper natural somatosensation on the fingertips.

Due to the limitations of these devices and the identified requirements, we targeted the development of a fully wearable and portable exoskeleton, that can control flexion and extension of fingers without hampering natural somatosensation on palms and fingertips, and as lightweight and soft as possible to enable comfortable and intensive use at home.

\section{B. Design}

Throughout the development phase of the device, we iteratively designed, tested and improved several concepts by integrating feedback acquired in functional tests with final users, healthcare therapists and physicians. All the tests and interviews were conducted at the SuvaCare-Clinique Romande de Radaptation (Sion, Switzerland), and at users' homes.

1) Design Requirements: According to the feedback we collected and based on previous literature research [4], [5], [17], the tasks identified as fundamental for regaining independence in ADL were: eating, drinking, toileting and using devices for communication (such as smartphones and computers). Many of these tasks can be performed through the combination of a restricted set of hand motions: power grasp (using all fingers, e.g. when holding glasses), precision grasp (using thumb, index and medium fingers, e.g. holding a pen) and lateral grasp (using thumb in opposition with respect to the other fingers, e.g. holding a toothbrush) [18]. During typical ADL, the ranges of motion for fingers' joints are usually in the range $[-10,100]^{\circ}$ and forces at the fingertips are within the range $[0,10] \mathrm{N}[8]$. These tasks do not impose strict requirements on the movements of single phalanxes, but rather require hand compliance in order to allow adaptation to different objects and shapes. Additionally, assistive devices should take less than 10 minutes to be donned or doffed, have a reduced weight to limit fatigue and their overall profiles, as well as the number of their hard parts, should be minimized for enabling comfortable use and transparent interactions with the environments surrounding the wearer [19].

2) Design Choices: For the mano device, we adopted a design that exploits bowden cables in a dual manner, as artificial tendons for motion transmission and as structural elements for the exoskeletal fingers. This choice had a double benefit. On the one hand, it allowed the remote placement 
of the actuation units with respect to the arm of the wearer, to the benefit of lightweight and low-profile structures on the limbs. On the other hand, it allowed to enable soft and fully compliant structures on the fingers, to the benefit of comfort and functionality. The exoskeletal fingers have been anchored on the dorsal side of the hand in order to free palm and fingertips from external mechanisms and to fully preserve natural somatosensation during interactions with external objects and tools.

Only one active degree of actuation for same-finger phalanxes is used. Similarly to state-of-the-art robotic grippers and to computational models for robust grasping [20], [21], the device exploits under-actuation and the physiological coupling between phalanxes in order to allow the objects under manipulation to shape the hand of the user. This induces natural and comfortable grasps without the independent actuation of all phalanxes or the intervention of complex closed-loop algorithms. The artificial tendons are used for bi-directional force transmission from the actuators, with compressive forces causing fingers flexion and tractive forces causing extension. Abduction and adduction of fingers cannot be actively controlled by the device; however, thanks to the passive autoalignment of the exoskeletal structures, users can freely exert these degrees of freedom without any limitation resulting from wearing the exoskeleton. The thumb, index and medium fingers are independently actuated, whereas the ring and little fingers are jointly actuated, enabling three types of grasps: power, precision and lateral.

Based on feedback from final users, we placed the actuation, control and energy storage units inside a chest-pack (rather than in a back-pack, as in similar state-of-the-art devices). This allows users sitting on a wheelchair to comfortably rest their backs and shoulders against the backseats of their wheelchairs, enabling comfortable use of the system for prolonged periods.

Finally, in order to limit the cost of the system, we only used off-the-shelf components and parts that can be sourced from typical shops for hobby modeling. The custom mechanical components were designed to be easily manufacturable by means of 3D printers and laser-cutters.

\section{Overview}

The mano system (see Figure 1) consists of two main parts, a chest-pack and actuated exoskeletal tendons-fingers. The chest-pack, wearable by means of adjustable belts, hosts the actuation, control and energy storage units. Its size is: $200 \times 210 \times 33$ [width $\times$ length $\times$ height] $\mathrm{mm}$. The artificial tendons consist of bowden cables (1.6 mm, Shimano, Japan), actuated by linear servomotors (L16 R, Actuonix Motion Devices Inc., Canada). Bowden cable sheaths $(5 \mathrm{~mm}$, Shimano, Japan) route the tendons from the actuators to the metacarpophalangeal (MCP) joints on the hand. The ends of the sheaths are fixed on the MCPs by means of soft elastic threads which loop around the fingers of the wearer. From the MCPs, the artificial tendons are routed and held in place onto the fingers by means of hooks-and-loops rings. The ends of the tendons are fixed using hooks-and-loop rings on the distal interphalangeal (DIP) joints, in order to free the fingertips from any external structure. No soft or hard structures are placed on the palm of the wearer.

The sheaths are held on the user's arm and wrist by means of two adjustable fabric bands and custom 3D printed sheathguides. The fabric bands are worn and unworn by means of commercial side-release buckles.

The control unit consists of a microcontroller (Arduino Mega 2560 R3, Arduino, Italy) that runs the firmware of the system, and of a custom printed-circuit board hosting a Bluetooth radio (HC-06, Guangzhou $\mathrm{HC}$, China) for wireless communication with external devices. The linear actuators provide a standard 3-wired interface for radio-controlled servomotors, allowing the implementation of closed-loop positioncontrol through the sending of pulse-width modulated (PWM) references by the microcontroller. The system is powered by a LiPo battery (H2B180, Hy-Line, Switzerland) that provides an autonomy of up to 3 hours in continuous operation mode (continuous opening and closing of five fingers).

The cover of the chest-pack and the sheath-guides were manufactured by means of 3D printing (EOSint P395, EOS, Germany and HP DesignJet 3D, Stratasys, US, respectively), the chest-pack backplate was cut from a $3 \mathrm{~mm}$ MDF panel using a laser-cutter (ProLF 90 W, Full Spectrum Laser, US).

The exoskeleton can be donned or doffed in less than seven minutes. The overall weight on the arm of the wearer is below $50 \mathrm{~g}$, the weight of the chest-pack is $930 \mathrm{~g}$.

Table I presents a comparison between the mano device and the state-of-the-art systems discussed in Section II-A. Compared to these devices, our approach has the unique advantage of enabling, within a single wearable and portable device, active control of fingers flexion and extension while preserving natural somatosensation on palms and fingertips.

\section{Mechanical characterization}

We characterized the mano system in terms of ranges of motion (ROM) and achievable forces at the fingertips. The characterization was performed on a human participant rather than on experimental test benches in order to allow capturing the non-linearities deriving from the soft and underactuated design of the device and from the non-linear couplings, during use, between the exoskeleton, the hand of the wearer and the external environment.

1) Ranges of motion: The ROM of the index finger were characterized in three different tasks: hand closing, hand opening and precision grasp of an object. For each task two different conditions were compared:

- No Exoskeleton: Hand motions were performed by the user, without assistance from the exoskeleton.

- Exoskeleton: Hand motions were performed by the exoskeleton, without contribution from the user.

During all the tasks and conditions, the subject was sitting on a chair, resting the right elbow on a table and holding the right hand at about $20 \mathrm{~cm}$ distance from the edge of the table. Each task was repeated 10 times (trials) for each condition. In the Exoskeleton trials, the device was worn on the right hand and the subject was instructed to restrain any voluntary hand motion in order to let the exoskeleton autonomously 
TABLE I

Comparison of the mano device (first row) to state-of-the-art hand exoskeletons. The force values refer to the sum of the maximum forces across all the fingertips. Note: Several references lacked information related to the weight of the device on forearms; in order to allow a fair comparison with our system, we supposed these devices to have approximately the same weight as ours.

\begin{tabular}{l} 
Device \\
\hline Randazzo et al. \\
Diftler et al. [10] \\
Ingvast et al. [11] \\
Nycz et al. [12] \\
In et al. [13] \\
Kang et al. [14] \\
Polygerinos et al. [15] \\
Varalta et al. [16]
\end{tabular}

implement the task. During the experiment, the beginning and the end of each trial were signaled by graphical cues on a computer screen. Subsequent trials were separated by rest periods of random durations (in the range [3,6] s). At each trial, the actuation of the device was started and terminated by a computer. Instructions on the tasks were provided before the beginning of the experiment.

The hand closing (opening) task consisted in finger flexion (extension), from a hand-open (hand-closed) position to a hand-closed (hand-opened) position; no objects were grasped for these tasks. The precision grasp task aimed at comparing ROM during a functional movement; in this case, the subject started from a hand-open position and performed a precision grasp by holding the thumb still and closing the index on a cylindrical object (a whiteboard marker, diameter: $1.75 \mathrm{~cm}$ ).

Motion of the joints was tracked using an eight-camera motion capture system (Vicon, Oxford Metrics Ltd, UK). According to the model proposed in Metcalf et al. [22], four spherical reflective markers for motion tracking were placed on the metacarpophalangeal (MCP), proximal interphalangeal (PIP) and distal interphalangeal (DIP) joints and on the distal head of the distal phalanx (FT) of the index finger (Fig. 2).

The three-dimensional (3D) joint trajectories were averaged across trials belonging to the same task and condition. Principal component analysis (PCA) was applied on the averaged trajectories to identify the planes where flexion/extension motions occurred and to discard spurious abduction/adduction movements. The 3D data was then projected on the two first principal components (which carried around $97 \%$ of the total variance), resulting in two-dimensional joints trajectories.

Figure 2A shows the trajectories of the MCP, PIP, DIP and FT joints, averaged across hand closing trials, for the No Exoskeleton and Exoskeleton conditions. The kinematics of the joints are similar across the two conditions during the first part of the task (checkpoint A to B), but they present differences in the last part (checkpoint B to D). Similar results were obtained for the hand opening task (results not shown). Based on the ROM of the joints, we computed the operational workspace of the device (Figure 2B). Results showed that the exoskeleton can cover more than $70 \%$ of the workspace observable in normal hand closing motions.

Figure 2C shows the trajectories of the MCP, PIP, DIP and FT joints, averaged across precision grasp trials. For this task, the ROM of the joints were similar across the

\begin{tabular}{c} 
Force [N] \\
\hline 20 \\
$\mathrm{n} / \mathrm{a}$ \\
12 \\
$\mathrm{n} / \mathrm{a}$ \\
20 \\
29.5 \\
40 \\
$\mathrm{n} / \mathrm{a}$
\end{tabular}

\begin{tabular}{cc}
\multicolumn{2}{c}{ Weight $[\mathbf{g}]$} \\
Main unit & Forearm \\
\hline & \\
930 & 50 \\
(see Forearm) & 770 \\
$\sim 650$ & $\sim 50$ \\
$\mathrm{n} / \mathrm{a}$ & $\sim 50$ \\
$\mathrm{n} / \mathrm{a}$ & 194 \\
1630 & $\sim 50$ \\
3300 & 285 \\
5000 & $\sim 50$
\end{tabular}

\begin{tabular}{cc}
\multicolumn{2}{c}{ Somatosensation } \\
Palm & Fingertips \\
\hline & \\
Full & Full \\
Absent & Absent \\
Absent & Limited \\
Absent & Absent \\
Absent & Absent \\
Absent & Limited \\
Full & Absent \\
Full & Absent
\end{tabular}

Exoskeleton and No Exoskeleton conditions, showing how the soft underactuation can enable functional and natural grasps.

2) Force at the fingertips: During this experiment, the subject was sitting on a chair, resting the right elbow on a table and holding the right hand at about $20 \mathrm{~cm}$ distance from the edge of the table with the hand's dorsal side facing the floor. An exoskeletal finger was worn on the index finger.

In subsequent steps, weights were attached to the FT of the index by means of a nylon rope, and the exoskeletal finger was actuated in order to lift them. At each step, a $50 \mathrm{~g}$ weight was added to the total weight, covering the overall range $[0,700] \mathrm{g}$. The subject was instructed to restrain any voluntary hand motion throughout the lifting phases in order to let the exoskeleton bear all the weight. For each step, the device performed 10 lifting motions. Instructions on the task were provided before the beginning of the experiment.

The maximum weight the exoskeletal finger was able to lift in a repeatable and reliable manner (i.e. completing, consistently across repetitions, 10 lifting motions) was $500 \mathrm{~g}$. For heavier weights, the exoskeleton tended to lose alignment with respect to the joints of the subject, resulting in inconsistent finger motions across repetitions.

These results translate into a maximum continuous force achievable at the fingertip of around $5 \mathrm{~N}$. For the exoskeleton configuration presented in Section II-B (with independentlyactuated thumb, index and medium and coupled-actuation for ring and little fingers), the maximum force across all the fingertips is around $20 \mathrm{~N}$.

\section{E. Functional tests with spinal-cord injured users}

The device was tested in typical ADL and functional tasks with two users who suffered from spinal cord injuries. Both users, 30-years-old S1 injured in May 2003 and 48-years-old S2 injured in December 1989, sustained complete lesions at the level C5-C6 and scored "A" in the ASIA scale (Complete injury-No motor or sensory function preserved in the sacral segments S4 or S5). S1 and S2 lack control of their lower limbs and have limited control of the upper limbs; they can perform reaching movements but have limited force at the proximal level and almost none in grasping and holding. Users gave their written consent to participate to the tests and the experimental protocols were approved by the local ethical committee.

All tests reported here were performed at users' homes. During the trials, we used a version of the device which 

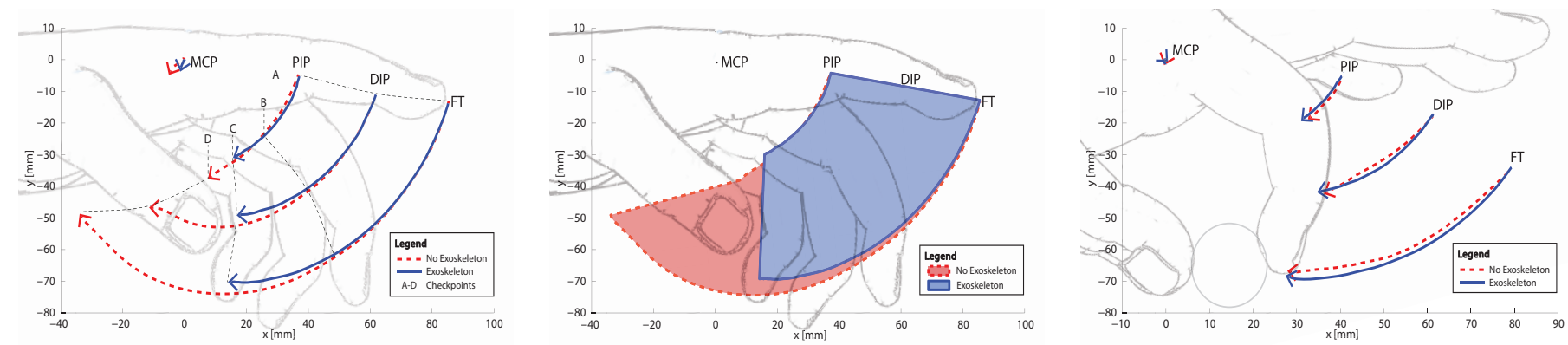

Fig. 2. Comparisons of kinematics of the index finger in No Exoskeleton and Exoskeleton conditions: (Left) Trajectories averaged across hand closing trials, (Middle) Operational workspaces, (Right) Trajectories averaged across precision grasp trials. The No Exoskeleton and Exoskeleton conditions are shown, respectively, in dashed-red and solid-blue. The origins correspond to the MCP joints.

equipped the thumb, index and medium finger modules. The ring and little fingers modules were not used because a three-fingered configuration already ensured stable and reliable grasps for both S1 and S2. Each test lasted around 1 hour. A total of 3 and 2 sessions were performed for S1 and S2, respectively. The tests consisted of two parts. In the first part, subjects grasped, displaced and released the following objects: a glass, a bottle, a fork, a knife, a salt-and-pepper dispenser, a highlighter, a sheet of paper, a pen, a lighter, and a toothbrush. In the second part, subjects performed exemplar ADL, namely: eating using cutlery, cleaning teeth by using a toothbrush and drinking from a glass of water. During the tests, users provided instructions on which fingers to actuate and to which degree to an experimenter, who was in turn controlling the device through a smartphone. The outcome of the tests was determined by the attainment or not of each of the tasks, as evaluated directly by the users.

Thanks to the mano system, S1 and S2 were able to regain some capabilities they had lost since their disabling accidents. In particular, we demonstrated the restoration of functions requiring both power and precision grasps. Subjects were capable to grasp and hold bottles weighing up to 1 $\mathrm{kg}$ by only using their fingertips (no weight was borne by their palms), autonomously drink from a glass full of water and use objects which require a coordinated exchange of forces between their fingers and the environment, such as using a knife and writing with a pen (see Supplementary Video). High satisfaction was reported by both subjects, who expressed a marked interest towards the possibility of using the system during ADL. The main features of interest were, according to them, the extremely reduced and lightweight profile of the system on their hands and the ability to sense the manipulated objects through the residual somatosensation at their fingertips. As reported by both users, these features allowed not to hamper the residual range of motions and forces of their shoulders, elbows and hands, and to confidently perceive when an exoskeleton-assisted grasp was safe and stable before performing a manual task. Overall, these results strongly support the usability of the mano exoskeleton in typical ADL.

\section{BRAIN-MACHINE INTERFACING}

In order to evaluate the usability of our exoskeleton within a neurorehabilitative framework, we exemplified its use in a brain-machine interface scenario.

Several studies have shown that manual tasks induce changes, with respect to baseline states, in the spectral power of sensorimotor cortex areas, for two characteristic frequency bands: $\mu([8,12] \mathrm{Hz})$ and $\beta([16,32] \mathrm{Hz})$. Such results have been confirmed in invasive [23] and non-invasive [24] studies. It has also been shown that passive hand mobilizations induce similar brain patterns as those observable in active manual tasks [25]. In a first analysis, we studied EEG rhythms during exoskeleton-induced motions in order to evaluate whether the device could elicit such characteristic brain patterns.

Similarly to what observed in active and passively-induced limb movements, it has also been shown that motor imagery (MI), the mental process of imagining and recalling body movements, induces changes in the spectral power of the $\mu$ and $\beta$ bands [26]. Indeed, the possibility of voluntarily modulating specific brain patterns through MI has been widely used as a control input for artificial devices [27]. In many of these studies control commands are delivered to devices on largely granular time-scales. It is however believed that, in neurorehabilitative scenarios, the possibility of providing patients with a real-time feedback about their ongoing cortical activity might be a more effective strategy for triggering brain plasticity and promoting motor recovery through practiceand-reward Hebbian processes [28], [29]. Despite continuous control of a hand exoskeleton through EEG-decoded MI and electrooculography (EOG) has been demonstrated [2], the feasibility of such type of control using EEG alone is still unknown. Indeed, while the use of EOG as a control channel could be a feasible option for assistive applications, it might not be an ecological approach for promoting motor recovery. Therefore, this study also aimed at investigating whether a hand exoskeleton could be continuously controlled by exploiting MI commands decoded only from EEG.

\section{A. Experimental design}

Nine right-handed subjects (mean age $23 \pm 5,2$ women), with no known history of neurological abnormalities or musculoskeletal disorders, participated in the experiment. During the experiment, subjects were sitting on a chair resting their elbows on a table and wore the exoskeleton on their right hands. EEG activity was recorded at $512 \mathrm{~Hz}$ from 16 active electrodes distributed over the scalp following the 10/20 in- 
ternational system (FZ, FC3, FC1, FCz, FC2, FC4, C3, C1, $\mathrm{Cz}, \mathrm{C} 2, \mathrm{C} 4, \mathrm{CP} 3, \mathrm{CP} 1, \mathrm{CPZ}, \mathrm{CP} 2, \mathrm{CP} 4)$, using a g.USBamp (gTec Medical Engineering $\mathrm{GmbH}$, Austria). Each participant was asked to perform four tasks:

- Resting (REST): subjects were asked to relax.

- Exoskeleton-induced hand motions (EXO): subjects were asked to relax while the exoskeleton closed their hands.

- Right hand motor imagery (MI): subjects performed kinesthetic motor imagery of closing their right hand; no exoskeleton-induced hand motions were performed.

- Exoskeleton-induced hand motions plus motor imagery (MIEXO): subjects performed kinesthetic motor imagery of closing their right hand while the exoskeleton closed their hands.

Tasks were repetitively performed in 4-s intervals (trials). Each subject performed at least 9 experimental blocks. Each block comprised 15 REST trials and 15 trials of one of the 3 other conditions. For the sake of minimizing any learning effect, the order of the trials within a block, as well as the order of the blocks was randomized for each subject. Approximately 50 trials per task were recorded for each subject. Before starting the experiment, the hands of the subjects were moved by means of the exoskeleton in order to allow them to get used to the EXO and MIEXO tasks. In the EXO and MIEXO trials, the exoskeleton was controlled by a computer to start from an open position and to reach hand closing at the end of the trial. Each experiment lasted, on average, 1.5 hours per subject. Instructions on the tasks were provided to each subject before the beginning of the experiment.

\section{B. Analysis}

EEG data were re-referenced using a laplacian spatial filter [30]. Then, the power spectral densities (PSD) of each electrode were extracted for the frequency range $[6,32] \mathrm{Hz}$, using a Short-Time Fourier Transform (computed in 1 s-long windows, with $93.75 \%$ overlap between consecutive windows). PSD values were used as features for all the analyses reported here. A feature is therefore the power spectral density of one electrode at one specific frequency.

First, we evaluated the existence of discriminable neural correlates of the exoskeleton task (EXO) with respect to the REST task. To this end, information on the discriminant power (DP) of features was assessed at the single-subject level via signed point-biserial correlation (signed- $r^{2}$ ).

We further analyzed whether the brain correlates of different tasks could be discriminated using pattern recognition techniques. Linear discriminant classifiers were built for the following comparisons: MI vs REST, EXO vs REST, MIEXO vs REST, and MIEXO vs EXO. The accuracy of each classifier was evaluated using 10-fold cross-validation. Classifiers were trained on the 10 most discriminant features (best $r^{2}$ score) of the respective training folds. Non-parametric signed rank statistical tests were performed for comparing results across comparisons at the population level, and the obtained p-values were Bonferroni-corrected. Additionally, all the accuracies were compared against the chance level at $95 \%$ confidence,

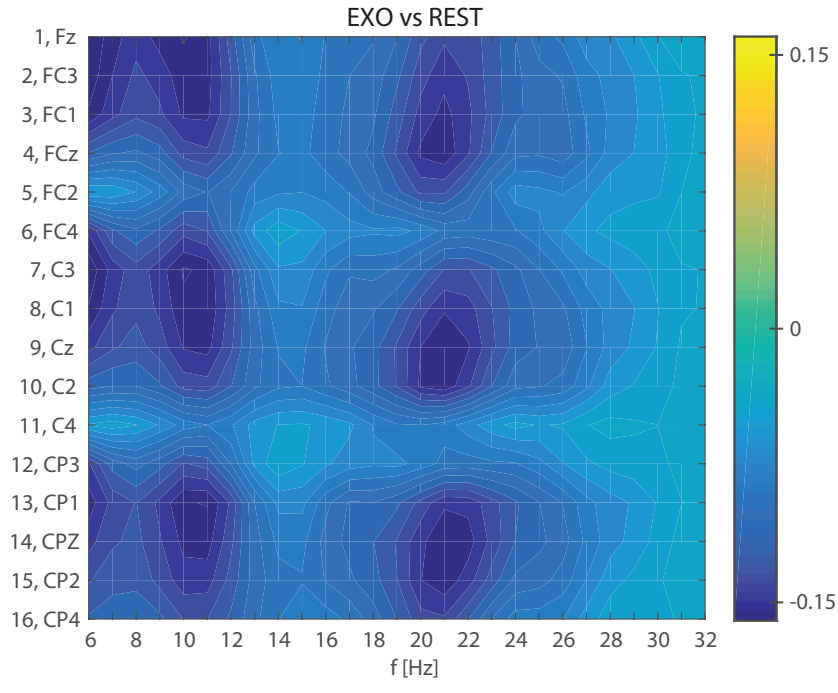

Fig. 3. Discriminant power (DP) map of PSD features for the EXO vs REST comparison, averaged across all subjects. DP are reported as signed pointbiserial correlation (signed- $r^{2}$ ). Negative values imply a desynchronization, i.e. the PSD in the EXO condition was lower than the PSD in the REST condition. $\mathrm{X}$ and $\mathrm{Y}$ axes represent, respectively, frequencies and electrodes.

estimated at $58 \%$ from the available number of trials $(N=50)$ using the binomial cumulative distribution [31].

Finally, we evaluated the most discriminant features for each comparison by using topological heatmaps. These maps were obtained by counting how many times features in the sensorimotor frequency bands were selected, for each EEG electrode, during the cross-validated construction of the respective classifiers.

\section{Neuroimaging of exoskeleton-induced hand motions}

Figure 3 reports the DP of PSD features for the EXO vs REST comparison, computed at the single subject level and, then, averaged across all subjects. Exoskeleton-induced hand motions led to a spectral power decrease compared to rest. Coherently with previous literature [25], [32], this pattern was mostly centered on electrodes located over the motor cortex. The desynchronization was largely bilateral and, mostly, confined over the $\mu$ and low- $\beta$ frequency bands. These results confirm that the exoskeleton can induce, in a repeatable manner across all subjects, EEG patterns of natural hand motions.

\section{Single-sample classification}

Figure 4A reports the classification accuracies for the four different comparisons. Accuracies were averaged across the 10-folds for each subject and, then, averaged across all subjects. On average, the performance of all the active conditions (MI, EXO, MIEXO) vs REST was above chance level: $63.02 \pm 5.91 \%, 69.64 \pm 5.74 \%$ and $72.19 \pm 6.57 \%$, respectively. Both EXO vs REST and MIEXO vs REST comparisons were significantly better than MI vs REST (two-tailed signed rank test, Bonferroni corrected $p<0.05$ ), indicating the potential use of exoskeleton-guided MI training as a way of improving the accuracy of the BMI system. 

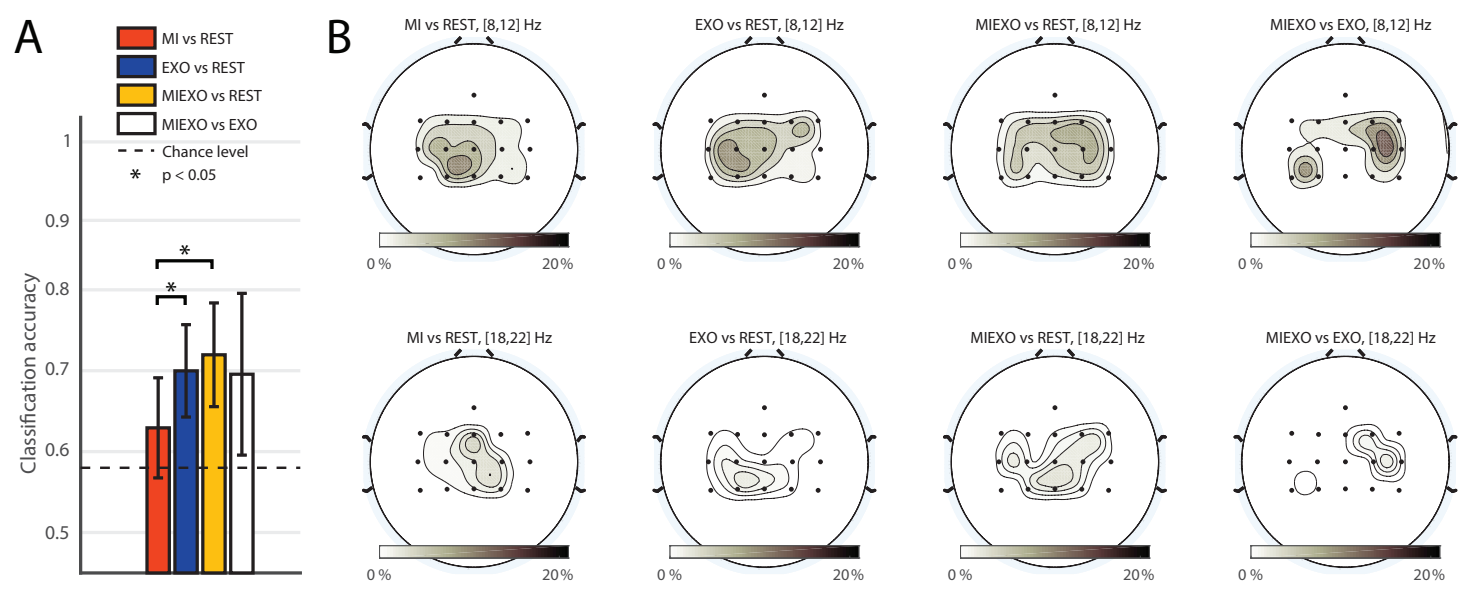

Fig. 4. Classification results averaged across all subjects. Panel A reports the accuracies for the four different comparisons. The dashed line indicates the chance level computed from a binomial distribution. Panel B shows the topographic heatmaps of features importance, averaged across the sensorimotor frequency bands ( $\mu$ : top row, low- $\beta$ : bottom row). The importance of a given feature is computed as the total number of times it was selected in the training folds of the respective cross-validation (a value of $100 \%$ indicates that the feature was selected in all the training folds, for all subjects).

Importantly, the MIEXO vs EXO classification accuracy was also significantly above chance level $(69.91 \pm 9.86 \%)$, indicating the feasibility of discriminating between passively induced movements and motor imagery coupled with exoskeleton-induced movements, a key issue in order to avoid biasing during continuous control of the device.

Figure 4B shows, in a topographic heatmap, the importance of features for the different comparisons. In line with literature [33], MI features were mostly selected over the contralateral motor cortex within the $\mu$ rhythm and, to a lower extent, in the $\beta$ frequency range. Interestingly, however, the MIEXO vs REST comparison also presented discriminant features over the ipsilateral hemisphere. This hemisphere is indeed the most important for differentiating MIEXO and EXO tasks. These results are in line with previous works which showed that active movements induce larger desynchronizations over ipsilateral regions than MI [33] and passive movements [32].

\section{DISCUSSION}

In this work, we presented a novel hand exoskeleton, mano, designed for use both in assistive and neurorehabilitative scenarios. The device has the unique advantage of combining, within a wearable and portable system, features as functionality, reduced bulk and comfortability, which could enable intensive use by users with motor disabilities in a wide range of settings, including home-based rehabilitation. The results obtained in the experimental characterizations and in tests with two users who suffered from spinal cord injuries strongly support the usability of the device in typical ADL. Due to its modular design, the device can be easily customized to meet the needs of a wider population of users, such as children affected by cerebral palsy, users who experienced cerebrovascular accidents or suffering from muscular weakness.

However, one of the main limitations of the current system is its limited usability by subjects suffering from hypertonia and spasticity, some of the most common consequences of motor disorders affecting the central and peripheral nervous systems. For these users, the continuous and unsupervised use of wearable robotic devices can be difficult or, even, counterproductive because of the resistance opposed by the hypertonic limbs. In such cases, robotic devices could be coupled to techniques aimed at reducing muscular tone (e.g. pharmaceutical drugs or functional electrical stimulation) for the sake of performing continuous motions and limiting the occurrence of limbs contractures due to excessive immobilization [5]. Other important issues to be considered for unsupervised use in ADL are also related to safety and acceptance [3]. Although our system does not achieve yet a level of maturity to address such problematics, we are continuing the tests with final users in order to identify the issues that could limit the adoption of such a device in home settings.

Our results also demonstrated that the hand mobilization provided by the system can reliably mimic natural hand movements, both in terms of physical motions and in terms of electrophysiological patterns elicited in the brain. In the future, we plan to perform equivalent evaluations with users who suffered from spinal cord injuries and brain stroke. Our findings could be possibly expanded by coupling exoskeletonprovided motions and brain imaging techniques for the sake of evaluating the integrity of the corticospinal pathways in users with disabilities.

Interestingly, we also showed that the use of the exoskeleton may have aided the subjects to better perform motor imagery tasks. However, further studies are needed to confirm whether the increase in performance is only due to the exoskeletoninduced motions or it is indeed due to improved embodiment through the rich and coherent feedback provided by the device. By showing that subject-dependent classifiers were able to uncover discriminable patterns between pure passive motions and exoskeleton-assisted motor imagery, this work started to tackle several open questions regarding continuous control exploiting only EEG signals. As an alternative, we have 
recently shown that other neural correlates could be used for the control of similar systems [34], [35].

Despite promising, many state-of-the-art techniques for human-machine interaction (HMI) still fail in meeting the high requirements that would allow unsupervised and reliable use at home. Nonetheless, recent progresses have shown great promises towards enabling transparent interfaces with the central and peripheral nervous systems through the combination of various electrophysiological signals (e.g. electroencephalography, electromyography, electrooculography) [36]. An interesting approach could aim at further combining these signals to voice commands or widespread smartphone-based interfaces. In this regard, it is important to notice that the mano exoskeleton is independent from the HMI control-layer. The user interface and the related control signals can be therefore adapted to the residual capabilities of the targeted subjects, their needs and the specific scenario of application.

Altogether, the results of this work show that the mano exoskeleton can provide an ecological way for inducing natural hand motions. By coupling the device to transparent control interfaces, the system could be used to translate motor intention into real hands motions, which in turn provide a rich and coherent proprioceptive feedback to the wearer, allowing to (re-)establish closed sensorimotor loops for its users. These approaches represent promising venues both for triggering neuroplasticity and recovery in people with motor disabilities and for enabling transparent human-machine interactions in daily scenarios.

\section{REFERENCES}

[1] C. Takahashi, L. Der-Yeghiaian, V. Le et al., "Robot-based hand motor therapy after stroke," Brain, vol. 131, no. 2, pp. 425-437, 2007.

[2] S. Soekadar, M. Witkowski, C. Gómez et al., "Hybrid EEG/EOGbased brain/neural hand exoskeleton restores fully independent daily living activities after quadriplegia," Science Robotics, vol. 1, no. 1, p. eaag3296, 2016.

[3] G. Turchetti, N. Vitiello, S. Romiti et al., "Why effectiveness of robotmediated neurorehabilitation does not necessarily influence its adoption," IEEE Reviews in Biomedical Engineering, vol. 86, no. 1, pp. 29-33, 2014.

[4] J. Krakauer, "Motor learning: its relevance to stroke recovery and neurorehabilitation," Current Opinion in Neurology, vol. 19, no. 1, pp. 84-90, 2006.

[5] B. Dobkin, "Strategies for stroke rehabilitation," Lancet Neurology, vol. 3, no. 9, pp. 528-536, 2004.

[6] R. Bos, C. Haarman, T. Stortelder et al., "A structured overview of trends and technologies used in dynamic hand orthoses," Journal of NeuroEngineering and Rehabilitation, vol. 13, no. 1, pp. 62-87, 2016.

[7] P. Heo, G. Gu, S.-j. Lee et al., "Current hand exoskeleton technologies for rehabilitation and assistive engineering," International Journal of Precision Engineering and Manufacturing, vol. 13, no. 5, pp. 807-824, 2012.

[8] P. Maciejasz, J. Eschweiler, K. Gerlach-Hahn et al., "A survey on robotic devices for upper limb rehabilitation," Journal of Neuroengineering and Rehabilitation, vol. 13, no. 5, pp. 807-824, 2014.

[9] C. Pacchierotti, S. Sinclair, M. Solazzi et al., "Wearable Haptic Systems for the Fingertip and the Hand: Taxonomy, Review, and Perspectives, in press," IEEE Transactions on Haptics, 2017.

[10] M. Diftler, C. Ihrke, L. Bridgwater et al., "RoboGlove: A Robonaut derived multipurpose assistive device," in International Conference on Robotics and Automation, 2014, pp. 78-84.

[11] M. Nilsson, J. Ingvast, J. Wikander et al., "The soft extra muscle system for improving the grasping capability in neurological rehabilitation," IEEE EMBS Conference, pp. 412-417, 2012.

[12] C. Nycz, M. Delph, and G. Fischer, "Modeling and design of a tendon actuated soft robotic exoskeleton for hemiparetic upper limb rehabilitation," IEEE EMBC Conference, pp. 3889-3892, 2015.
[13] H. In, B. Kang, M. Sin, and K.-J. Cho, "Exo-Glove: Soft wearable robot for the hand using soft tendon routing system," IEEE Robotics \& Automation Magazine, 2015.

[14] B. Kang, H. Lee, H. In et al., "Development of a polymer-based tendondriven wearable robotic hand," IEEE ICRA Conference, pp. 3750-3755, 2016.

[15] P. Polygerinos, Z. Wang, K. Galloway et al., "Soft robotic glove for combined assistance and at-home rehabilitation," Robotics and Autonomous Systems, vol. 73, pp. 135-143, 2015.

[16] V. Varalta, A. Picelli, C. Fonte et al., "Effects of contralesional robotassisted hand training in patients with unilateral spatial neglect following stroke: a case series study," Journal of NeuroEngineering and Rehabilitation, vol. 22, no. 1, pp. 97-105, 2014.

[17] K. Anderson, "Targeting recovery: priorities of the spinal cord-injured population," Journal of Neurotrauma, vol. 8, no. 8, pp. 741-754, 2009.

[18] M. Santello, M. Flanders, and J. Soechting, "Postural hand synergies for tool use," Journal of Neuroscience, vol. 18, no. 23, pp. 10 105-10 115, 1998.

[19] B. Brewer, S. McDowell, and L. Worthen-Chaudhari, "Poststroke upper extremity rehabilitation: A review of robotic systems and clinical results," Topics in Stroke Rehabilitation, 2007.

[20] R. Balasubramanian and A. Dollar, "A framework for studying underactuation in the human hand," Annual Meeting of the American Society, pp. 89-91, 2010.

[21] A. Bicchi and V. Kumar, "Robotic grasping and contact: A review," IEEE ICRA Conference, vol. 1, pp. 348-353, 2000.

[22] C. Metcalf, S. Notley, P. Chappell et al., "Validation and application of a computational model for wrist and hand movements using surface markers," IEEE Transactions on Biomedical Engineering, vol. 14, no. 6, pp. 22-44, 2007.

[23] M. Branco, Z. Freudenburg, E. Aarnoutse et al., "Decoding hand gestures from primary somatosensory cortex using high-density ECoG," Neurolmage, vol. 147, pp. 130-142, 2017.

[24] S. Hétu, M. Grégoire, A. Saimpont et al., "The neural network of motor imagery: An ALE meta-analysis," Neuroscience and Biobehavioral Reviews, vol. 37, no. 5, pp. 930-949, 2013.

[25] T. Mima, N. Sadato, S. Yazawa et al., "Brain structures related to active and passive finger movements in man," Brain, vol. 122, no. 10, pp. 1989-1997, 1999.

[26] A. Solodkin, P. Hlustik, E. Chen et al., "Fine modulation in network activation during motor execution and motor imagery," Cerebral Cortex, vol. 14, no. 11, pp. 1246-1255, 2004.

[27] J. d. R. Millán, R. Rupp, G. Müller-Putz et al., "Combining braincomputer interfaces and assistive technologies: State-of-the-art and challenges," Frontiers in Neuroscience, vol. 4, pp. 162-177, 2010.

[28] A. Ramos-Murguialday, D. Broetz, M. Rea et al., "Brain-machine interface in chronic stroke rehabilitation: A controlled study," Annals of Neurology, vol. 74, no. 1, pp. 100-108, 2013.

[29] B. Dobkin, "Brain-computer interface technology as a tool to augment plasticity and outcomes for neurological rehabilitation," The Journal of Physiology, vol. 579, no. 3, pp. 637-642, 2007.

[30] D. McFarland, L. McCane, S. David et al., "Spatial filter selection for EEG-based communication," Electroencephalography and Clinical Neurophysiology, vol. 103, no. 3, pp. 386-394, 1997.

[31] S. Waldert, H. Preissl, E. Demandt et al., "Hand movement direction decoded from MEG and EEG," Journal of Neuroscience, vol. 28, no. 4, pp. 1000-1008, 2008.

[32] C. Weiller, M. Jüptner, S. Fellows et al., "Brain representation of active and passive movements," NeuroImage, vol. 4, no. 2, pp. 105-110, 1996.

[33] D. McFarland, L. Miner, T. Vaughan et al., "Mu and beta rhythm topographies during motor imagery and actual movements," Brain Topography, vol. 12, no. 3, pp. 177-186, 2000.

[34] L. Randazzo, I. Iturrate, R. Chavarriaga et al., "Detecting intention to grasp during reaching movements from EEG," in IEEE EMBC Conference, 2015, pp. 1115-1118.

[35] I. Iturrate, R. Leeb, R. Chavarriaga et al., "Decoding of two hand grasping types from EEG," in 6th International BCI Meeting, 2016, p. 66.

[36] D. Borton, S. Micera, J. d. R. Millán, and G. Courtine, "Personalized neuroprosthetics," Science Translational Medicine, vol. 5, no. 210, p. 210rv2, 2013. 